\title{
Ambulant allein ist keine Antwort
}

\section{Anna Sax}

lic. oec. publ., MHA, Mitglied der Redaktion

\author{
Eine Förderung der ambulanten Medizin ist für sich allein keine Patentlösung für \\ die Probleme des Gesundheitswesens. Mit einer einseitigen Erhöhung der ambu- \\ lanten Tarife würden Anreize zur Mengenausweitung geschaffen und der Anstieg \\ der Krankenkassenprämien weiter beschleunigt. Wenn der Grundsatz «ambulant \\ vor stationär» sozialverträglich umgesetzt werden soll, sind flankierende Mass- \\ nahmen notwendig.
}

\begin{abstract}
La médecine ambulatoire n'est pas une réponse en soi

Encourager la médecine ambulatoire n'est pas en soi une solution miracle à tous les problèmes du domaine de la santé. En augmentant de manière unilatérale les tarifs des prestations ambulatoires, les médecins seraient incités à fournir davantage de prestations et les primes d'assurance-maladie continueraient leur progression. Par conséquent, si l'on veut appliquer le principe "l'ambulatoire avant le stationnaire», des mesures d'accompagnement sont nécessaires.
\end{abstract}

Michael Stamm weist zu Recht darauf hin, dass noch immer viele Gesundheitsleistungen stationär erbracht werden, die sinnvollerweise in einem ambulanten Setting möglich wären. Die neue Spitalfinanzierung über SwissDRG hat nicht nur Anreize geschaffen, Patientinnen und Patienten vermehrt ambulant zu betreuen, sondern wirkt, wie von Herrn Stamm beschrieben, teilweise auch in die entgegengesetzte Richtung. Einiges deutet darauf hin, dass chirurgische Eingriffe unter SwissDRG den Spitälern gute Erträge einbringen und ihre Häufigkeit entsprechend zugenommen hat, auch wenn ein solcher Zusammenhang bisher nicht mit Studien belegt ist. Trotzdem: Der Trend geht nicht erst seit Einführung der Fallpauschalen in Richtung (spital-)ambulante Versorgung, wie aus den Kostenstatistiken des Bundes ersichtlich ist [1].

\section{Nicht die Kosten sind kritisch ...}

Die Kostenentwicklung im Gesundheitswesen insgesamt ist weniger dramatisch, als sie in den Medien und in der Politik häufig dargestellt wird. Der Anstieg des Anteils der Gesundheitsausgaben am Bruttoinlandprodukt (BIP) ist kein Naturgesetz, sondern widerspiegelt auch die Wirtschaftsentwicklung: In wirtschaftlich erfolgreichen Zeiten, etwa in den Jahren 2006-2008, sank der Anteil der Gesundheitsausgaben am BIP auf unter 10\%, um nach Eintritt der Finanzkrise wieder auf knapp 11\% anzusteigen. Ähnliche Entwicklungen sind auch in unseren Nachbarländern Deutschland und Frankreich zu beobachten. Bei einer positiven Betrachtung könnte man sagen, dass der Gesundheitssektor eine Wirtschaftsbranche ist, die auch in Krisenzeiten ein konstantes Wachstum aufweist. Ob dieses Wachstum "gesund» oder «ungesund» ist, hängt von der umstrittenen Frage ab, welcher Nutzengewinn damit verbunden ist. Die höchsten Kosten fallen in der letzten Lebensphase

Ob dieses Wachstum "gesund" oder "ungesund" ist, hängt von der umstrittenen Frage ab, welcher Nutzengewinn damit verbunden ist.

an. Deshalb müssen wir uns für die kommenden Jahrzehnte in der Tat auf einen stärkeren Anstieg der Gesundheitsausgaben gefasst machen, wenn die «Babyboomers» der geburtenstarken Jahrgänge ins hohe Alter kommen und damit die Zahl der Todesfälle zunehmen wird.

\section{... sondern die Finanzierung}

Das Problem liegt weniger beim Anstieg der Gesundheitsausgaben als bei ihrer Finanzierung: Es spielt verteilungspolitisch eine wichtige Rolle, wie viele Gesundheitsleistungen wir aus der eigenen Tasche, über direkte Steuern oder über Krankenkassenprä- 


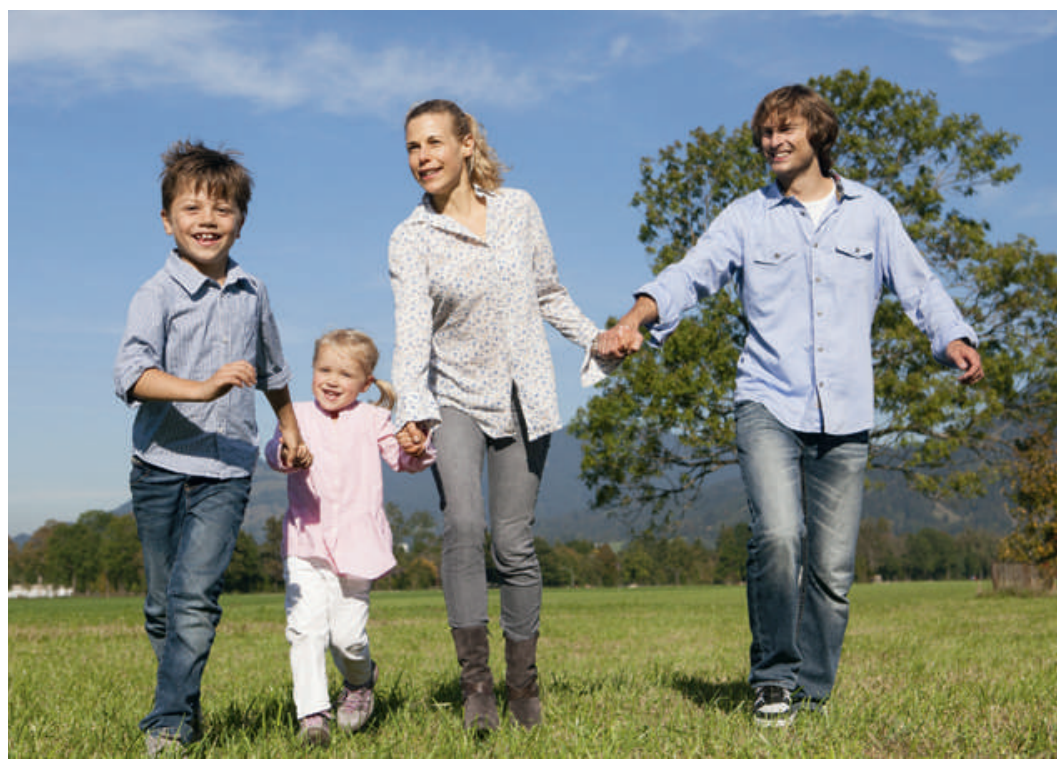

Eine vierköpfige Familie des unteren Mittelstands muss bis zu 16\% ihres verfügbaren Einkommens für Krankenkassenprämien ausgeben. Mehr und teurere ambulante Behandlungen würden zu einem weiteren Prämienanstieg führen.

mien finanzieren. Der Anteil der selbst finanzierten Leistungen und Kostenbeteiligungen ist in der Schweiz mit 25\% höher als in allen anderen OECDLändern. Der steuerfinanzierte Anteil liegt bei 20\%, über die obligatorische Krankenversicherung bezahlen wir 36\%. Die restliche Finanzierung erfolgt über andere Sozialversicherungen, Privatversicherungen und Ergänzungsleistungen zu AHV und IV. Wegen der

\section{Werden ambulant erbrachte Leistungen im} Vergleich zu den stationären besser abgegolten, schlägt dies voll auf die Grundversicherung.

Kopfprämien in der Krankenversicherung ist die Belastung der Haushalte mit kleinen und mittleren Einkommen - sofern sie über der Schwelle zur Prämienverbilligung liegen - überaus hoch. Je nach Kanton gibt eine vierköpfige Familie des unteren Mittelstands bis zu $16 \%$ ihres verfügbaren Einkommens für Krankenkassenprämien aus [2]. Der Anstieg der Kosten und damit der Prämien in der obligatorischen Grundversicherung muss als dramatisch bezeichnet werden: Während die Löhne zwischen 2000 und 2012 um $18 \%$ und die Konsumentenpreise um knapp 9\% stiegen, lag der Anstieg der Krankenkassenprämien in der gleichen Zeitspanne bei über 80\%. Die hohen und weiter steigenden Kopfprämien und der grosse Anteil selbst bezahlter Kosten werden, in Kombination mit den Sparmassnahmen in den Kantonen, über kurz oder lang zu einer sozialen Zerreissprobe führen.

\section{Finanzierungsschlüssel muss angepasst werden}

Hier kommt es nun zum Konflikt mit der Forderung nach einer weiteren Verschiebung von Leistungen vom stationären in den ambulanten Bereich, denn das überdurchschnittliche Kostenwachstum bei den ambulanten Spitalleistungen ist der wichtigste Treiber für den Anstieg der Krankenkassenprämien. Während bei einem stationären Spitalaufenthalt der Kanton (und damit die Steuerzahlenden) die Hälfte der Rechnung begleicht, geht eine ambulante Behandlung voll auf Kosten der Krankenkasse. Werden also ambulant erbrachte Leistungen im Vergleich zu den stationären besser abgegolten, schlägt dies voll auf die Grundversicherung durch und verschärft damit das Problem des Prämienanstiegs. Nur mit einer Angleichung des Finanzierungsschlüssels über die ganze Versorgungskette hinweg kann verhindert werden, dass ein weiteres Wachstum des ambulanten Bereichs die Prämien in die Höhe treibt. Eine monistische Finanzierung löst das Problem nicht. Wichtig ist vielmehr, dass die Höhe der Kantonsbeiträge insgesamt beibehalten wird.

In dieser Frühjahrssession behandelt der Ständerat einen Vorstoss, der vom Nationalrat bereits angenommen wurde. Er fordert die Angleichung des Finanzierungsschlüssels wenigstens für stationäre und ambulante Spitalleistungen.

Die Stärkung der ambulanten Versorgung mag medizinisch Sinn machen. Wenn sie auch ökonomische und sozialpolitische Vorteile bringen soll, dann sind höhere Abgeltungen für ambulante Leistungen ohne eine Veränderung der Rahmenbedingungen kontraproduktiv. Durch Einzelleistungstarife verursachte Fehlanreize müssten beseitigt, die Kooperation zwischen Leistungserbringern und Berufsgruppen müsste gefördert werden, z.B. mit bereichsübergreifenden Pauschalen, wie bei Herrn Stamm in einer Fussnote erwähnt. Was wir seit einigen Jahren erleben, ist ein massiver Ausbau der spitalambulanten Behandlungen ohne entsprechende Einsparungen im stationären Sektor, verbunden mit einer zunehmend regressiveren Finanzierung der Gesundheitsausgaben. Mit anderen Worten: Die Gesundheitsfinanzierung führt zu einer Umverteilung von den armen zu den reichen Haushalten.

\section{Referenzen}

1 www.bfs.admin.ch $\rightarrow$ 14-Gesundheit $\rightarrow$ Kosten, Finanzierung

2 B,S,S. Volkswirtschaftliche Beratung AG, im Auftrag des BAG (2012): Monitoring 2010. Wirksamkeit der Prämienverbilligung. 